\title{
ALBA Y ALIANZA DEL PACÍFICO: ¿CHOQUE DE INTEGRACIONES?
}

\author{
ALBA and the Pacific Alliance: A Clash of integrations?
}

\author{
Gilberto Aranda* \\ Sergio Salinas**
}

\section{RESUMEN}

A partir de la segunda mitad de siglo XX en América Latina se desarrolló una tendencia hacia la integración. Y aunque en sus inicios se basaba en el modelo europeo, es indiscutible que ha recorrido un derrotero basado en un pensamiento propio y singular. A partir del siglo XXI, estas experiencias tienen afinidades políticas y objetivos compartidos confirmando la idea que la identidad regional es también socialmente construida. Por cierto que implican conflictos de liderazgo, por ejemplo entre Estados Unidos y Brasil, institucionalizados en propuestas como el ALCA o la Unión Sudamericana de Naciones (UNASUR) o estrategias de desarrollo que se plasman en la formación de diversos bloques. En dos de estas alianzas, el ALBA y la Alianza del Pacífico, es más nítido visualizar el conflicto ideo-programático. El ALBA sitúa en el núcleo de su propuesta de integración la deuda social, lo que le permite desplegar recursos de poder blando directo. En tanto que la Alianza del Pacífico

\footnotetext{
${ }^{1}$ Este trabajo es parte del proyecto Fondecyt de Iniciación No 11130638 "La influencia de las corrientes partidarias y los liderazgos nacionales en la arquitectura institucional regional”. Un avance fue presentado en en el XXXVI Congreso Internacional de Americanística de Perugia, en mayo de 2014 bajo el título "Tres versiones del regionalismo sudamericano".

* Instituto de Estudios Internacionales, Universidad de Chile. Santiago, Chile / Instituto de Estudios Internacionales, Universidad Arturo Prat. Iquique, Chile. Correo electrónico: garanda@uchile.cl

** Doctor en Estudios Latinoamericanos de la Universidad de Chile. Santiago, Chile. Correo electrónico: ssalinas@uchile.cl
}

Artículo recibido el 14 de marzo de 2014. Aceptado el 7 de octubre de 2014. 
responde al modelo de regionalismo abierto aunque renovándolo mediante el reemplazo de la competencia por la cooperación en el acceso a los mercados de Asia Pacífico.

Palabras clave: Integración, regionalismo, relaciones internacionales, regionalismo abierto.

\begin{abstract}
Since the second half of the twentieth century, Latin America has developed a tendency to integration. And though its beginning was based on the European model, it is undeniable that its path has been based on a singular and own one. Then, in the twenty first century, these experiences have had political similarities and shared objectives, confirming the idea that regional identity is also socially constructed. Certainly, this view implies conflicts that deal with leadership. An example of this is the FTAA Agreement (Free Trade Area of the Americas Agreement), which has institutionalized proposals between the United States and Brazil, or the USAN (Union of South American Nations) or the different development strategies that are presented in the formation of different blocks. In two of these alliances, the ALBA and the Pacific Alliance, it is easier to visualize an ideological and programmatic conflict. The ALBA has a concrete integration goal, in which the social debt will allow to display the direct soft power resources. Meanwhile, the Alianza del Pacífico will respond to the open regionalism model, although it will have to be renewed by replacing the cooperative competence in the access of the Asian Pacific markets.
\end{abstract}

Keywords: Integration, regionalism, international relations, open regionalism.

\title{
INTRODUCCIÓN: LOS MOMENTOS DEL REGIONALISMO
}

Desde la segunda mitad de siglo XX en América Latina se desarrolló una tendencia hacia una integración. Si bien existen antecedentes decimonónicos, con el proyecto de Bolívar y las propuestas culturales de Andrés Bello, entre otras, es en la centuria pasada el espacio temporal de despliegue de opciones políticas concretas. Tal como señala Silva (2012:2) pueden distinguirse tres etapas o momentos que no necesariamente replican el proceso de integración europeo con sus fases sucesivas de cooperación económica, área de libre comercio y unión aduanera, adición de políticas económicas orientadas a generar un mercado común y la libre circulación de personas, bienes y capitales, y el culmen de una unión económica y monetaria (Balassa, 1964). En síntesis una experiencia que se fundamenta teóricamente en los aportes del funcionalismo, el neo-funcionalismo, y del desborde por área (spillover), más el recetario propuesto por la Comisión Económica para América Latina y el Caribe (CEPAL). Y aunque, particularmente en sus inicios la integración latinoamericana tuvo un impulso en la observación del 
modelo europeo, es indiscutible que ha recorrido un derrotero propio basado en un pensamiento propio y singular (Bernal-Meza, 2013: 47).

Los tres momentos aludidos obedecen a la creación de instituciones o bloques: El primero, signado por el proteccionismo, con el Mercado Común Centroamericano (MCCA) de 1960, el Pacto Andino en 1969 y la Comunidad del Caribe (CARICOM) de 1973. El segundo, que ha sido llamado neoregionalismo en virtud de renovar el impulso hacia una convergencia regional en torno a la estrategia del regionalismo abierto, supuso la transformación de algunos como el MCCA en SICA (Sistema de Integración Centroamericano) en 1991, y el Pacto Andino en Comunidad Andina de Naciones (CAN) en 1997. Adicionalmente surgen otros como la Asociación de Estados del Caribe en 1994 que reúne a estados insulares y continentales, el propio Mercado Común del Sur (MERCOSUR) en 1991 y el Grupo de los Tres que integraba México, Colombia y Venezuela en 1994.

Complementariamente, la creación de un mercado global unificado implicó la generación de normas multilaterales consensuadas de la OMC (Organización Mundial del Comercio) a lo que se sumaron los acuerdos de regionalización, tales como la Asociación Latinoamericana de Libre Comercio creada en 1961, gravitaron sobre los diversos bloques regionales latinoamericanos, particularmente, MERCOSUR, CAN, SICA y CARICOM, sin medrar la trayectoria de la ALADI (Asociación Latinoamericana de Integración fundada en el Tratado de Montevideo de 1980) en orden a generar Acuerdos de Complementación Económica (ACE) ${ }^{2}$.

Sin embargo, en la práctica el proceso de institucionalización de ciertos acuerdos, tales como las uniones aduaneras o zonas amplias de libre comercio como la iniciativa Área de Libre Comercio de las Américas (ALCA), y toda la dinámica integracionista regional en general no alcanzó los resultados esperados.

En el caso de MERCOSUR -unión aduanera imperfecta con un arancel externo común que va del 0 al $20 \%$-, cosechó inicialmente éxitos notables al incorporar a economías del tamaño de Brasil y Argentina en un mismo espacio, pero que ha experimentado crisis recurrentes por la autonomía cambiaria y monetaria de sus miembros mayores, y la permanente demanda de los estados de menor tamaño, Uruguay y Paraguay de ser inconsultos en ciertas decisiones del bloque.

La CAN ha sufrido los mayores traspiés, particularmente cuando algunos de sus miembros optaron por seguir estrategias económicas autónomas del bloque,

\footnotetext{
${ }^{2}$ Por lo general apuntaron a que los países firmantes accedieran al libre comercio en parte sustantiva de su intercambio por medio de la fijación de un cronograma, abriéndose a otras áreas de la cooperación y liberalización económica como el sector servicios, la promoción y protección de inversiones mutuas, la homogenización de las reglas técnicas y fitosanitarias, y la construcción de infraestructura.
} 
como Perú y Colombia que signaron tratados de Libre Comercio (TLC) de modo directo y no como miembros de la Comunidad. La respuesta de la Venezuela chavista fue la salida del bloque en 2006 y su ingreso al MERCOSUR. Hoy no se descarta que Bolivia siga las mismas aguas.

La CARICOM con una estructura más elástica catalizó el ingreso de sus estados parte a un área comunitaria de programas, que van desde un mercado común hasta la coordinación de políticas exteriores y cooperación sectorial.

El SICA siguió el derrotero de la una unión aduanera, antes de acometer las negociaciones directas con otros bloques como la Unión Europea, a la que se sumaron República Dominicana y Belice. La viabilidad del acuerdo ha sido garantizada por el Banco Interamericano de Desarrollo (BID), que ha puesto el énfasis en el desarrollo de acciones nacionales en el ámbito de la energía y la conectividad.

Mayores logros, aunque todavía insuficientes, exhibieron los esquemas ACE en ALADI y las redes de TLC como los que ha suscrito Chile, Perú, Colombia, dentro y fuera de la región. Lo anterior permite a Hernández (2013: 118), caracterizar la década de los noventa del siglo XX como signada por un proceso diádico de integración regional entre los países de la región y la apertura bilateral y multilateral. El así llamado Consenso de Washington se impuso con su decálogo de reformas: disciplina fiscal, cambio de las prioridades del gasto público; reforma fiscal, tipos de interés, tipos de cambio, liberalización comercial, política de apertura respecto a la inversión extranjera directa, política de privatizaciones, política de desregulación, derechos de propiedad (Casilda 2004: 23).

Después de una década de esta experiencia surgieron voces críticas. Desde un punto de vista escéptico del denominado Consenso de Washington, un mejor desempeńo de la región se sostendría en el "desarrollo de nuevos servicios", el complejo reto de competitividad con economías pequeñas y un "reposicionamiento estratégico global" (Payton, 2009: 145 y 153). El giro apuntó a reemplazar ciertas premisas del referido Consenso de Washington por un nuevo espíritu que priorizara una acción latinoamericana más directa, en que la apertura comercial fuera observada a partir de los parámetros domésticos, recibiendo críticamente el tipo de recetas como las sugeridas en los años 90 por el Fondo Monetario Internacional (FMI), y el Banco Mundial. Después de la convergencia de los noventa, el nuevo milenio significó la irrupción de la divergencia creciente de la región (Malamud, 2009) o la fragmentación subregional de la integración latinoamericana en torno a tres ejes: aperturista, revisionista y anti-sistémico (Briceño, 2013). Otra forma de aproximarse a la divergencia o fragmentación regional de principios del siglo XXI es observando la emergencia de alianzas menos convencionales -es decir más distantes de la clásica experiencia de integración europea-, sin cesión de soberanía en ningún nivel, o con escasos trazos de juridicidad vinculante, espacios en que el 
criterio de proximidad geográfica abdica en favor de afinidades políticas y objetivos compartidos confirmando la idea que la identidad regional es también socialmente construida (Hettne y Söderbaum, 2000:7) -nacionalmente diríamos más bienpor medio de un proceso de regionalismo que transforma el espacio geográfico en una subjetividad activa. Aquí es necesario distinguir conceptos cuyo epicentro radica en la idea de región (Morandé, 1999:3). En primer lugar, región como cuño relativo a una experiencia histórica común, un sentimiento compartido de problemas comunes entre un grupo de poblaciones que generan la identidad. Enseguida, el momento cuando los vínculos se estrechan se bosqueja una frontera al interior de la cual las interacciones son más intensas que aquellas con el mundo circundante, referido a una regionalización. Posteriormente se institucionaliza el proceso, por medio del cual la región adquiere un sentido de configuración normativa al dotarse de reglas del juego precisas al interior de la región, lo que le permite acceder a un franco regionalismo. En esta misma línea con relación a los Estados, Atkins refiere a Thomas para afirmar que un subsistema es "un conjunto de Estados geográficamente próximos que interactúan regularmente y comparten hasta cierto punto un sentido de identidad regional, reconocido por los actores exteriores" (Atkins, 1991). Bernal-Meza agrega que son las diferencias que configuran las identidades específicas las que pasan a ser las unidades de análisis por los investigadores (Bernal-Meza, 2011:13). En esta formulación adquieren notoriedad cualidades como proximidad e intensidad de una relación que se evalúa construida antes que dada espontáneamente. Hurrell avanza otro paso para proponer que las regiones son productos de actores políticos que consideran crucial la idea de una región (Hurrel, 2007) .

Por cierto que estos procesos de construcción regional implican conflictos de liderazgo (Nolte, 2006: 6), de estrategias de desarrollo (Actis, 2014: 74 y Busso, 2014: 12) y los caminos dispares de inserción global (Malamud, 2009: 136) que se plasman en la formación de los tres ejes referidos: aperturista, revisionista, antisistémico (Briceño, 2013), en otras palabras los bloques de Alianza del Pacífico, MERCOSUR y ALBA. Aunque estas diferencias se advierten en la competencia entre el liderazgo norteamericano en el hemisferio americano, expresado en iniciativas tales como la Alianza para el Progreso en los sesenta o el Área de Libre Comercio de las Américas hacia el cierre del milenio, y el prominente papel de Brasil en Sudamérica plasmado en el proyecto de la Unión Sudamericana de Naciones (UNASUR) que no contempla la participación de Estados ajenos a la subregión. No obstante, el conflicto ideo-programático es mucho más nítido en las estrategias y prioridades enarboladas por las dos alianzas articuladas en el escenario

\footnotetext{
${ }^{3}$ Hurrel señala la existencia de cinco tipos de procesos no lineales: regionalización, cohesión regional e identidad, cooperación interestatal, integración liderada por el Estado y consolidación regional.
} 
latinoamericano del siglo XXI: la Alternativa Bolivariana para las Américas (ALBA) y la Alianza del Pacífico. Ambas consagran la divergencia latinoamericana respecto de sus proyectos por lo que pasamos a evaluarlas a continuación.

\section{EL ALBA}

En el caso del ALBA enunciada en diciembre de 2001 por el presidente Chávez durante la III reunión de la Asociación de Estados del Caribe realizada en la isla Margarita, con un planteamiento para trascender el liberalismo económico mediante la adopción de estrategias sociales, políticas y seguridad comunes basadas en la potencia energética venezolana signada por la diplomacia

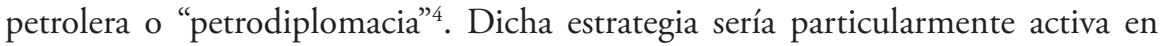
Centroamérica y el Caribe a partir de dicho momento. Originalmente recibió el respaldo cubano perfilándose en un marco para la acción posneoliberal sobre la base del cuestionamiento al modelo anterior y que sostiene nuevos valores, prácticas e instituciones incipientes que superan el horizonte sudamericano para instalarse también en América Central y el Caribe.

Durante la Cumbre de las Américas de Mar de Plata de noviembre de 2005 la posición de rechazo al ALCA se profundizó proponiéndose en su lugar la construcción de un polo regional orientado a la profundización de la cooperación, especialmente en los ámbitos financieros, energéticos, y telecomunicacionales, para mejorar los niveles de justicia social, aunque reteniendo los márgenes de autonomía económica y política tradicionales de los Estados (Gardini, 2010: 20). De esta manera "se retoman las ideas de integración con el fin de promover una gobernanza autónoma, inserción internacional, la modernización y la reducción de la dependencia" (Oyarzún, 2012: 6). En este punto es posible establecer que la experiencia ALBA funge como catalizador de mayores rangos de autonomía del bloque respecto a la potencia global dominante. Se trata del concepto puigiano de la autonomía heterodoxa referida al estadio en que las elites nacionales aceptando el liderazgo estratégico de la potencia dominante, divergen de la misma en: a) el modelo de desarrollo interno, b) los vínculos internacionales que no sean

\footnotetext{
${ }^{4}$ Según Quintanar los pasos más contundentes en el ámbito de esta cooperación energética ha sido la propuesta de PETROAMÉRICA en el año de 2003. La idea era que los Estados partes crearan una empresa intergubernamental regional en la que convergerían tres mecanismos de alcance subregional: PETROCARIBE, para el Caribe y Centro América, PETROSUR para el área de Brasil y el Cono Sur, y PETROANDINA para la Comunidad Andina. Mediante el incentivo de proyectos de inversión se ha propiciado el aumento del valor agregado del petróleo crudo y el gas, además de la atención a la explotación de subproductos petroquímicos necesarios para fomentar el desarrollo sostenible de la región (Quintanar, 2012: 242).
} 
globalmente estratégicos, c) la distinción entre los intereses nacionales de la potencia dominante y los intereses estratégicos del conjunto rechazando cualquier imposición dogmática al respecto (Puig, 1984: 63). Ahora bien, aunque es posible establecer que en el discurso el chavismo suele ir más lejos y plantear francamente la secesión -estadio también contemplado por Puig, que conlleva el riesgo de potencial regreso a una situación para-colonial si el Estado que enarbola la secesión no tiene la capacidad de alcanzarla-. Sin embargo, en la práctica Venezuela no ha modificado su estrecha relación comercial con Estados Unidos, como principal comprador de petróleo y socio comercial de Caracas (Gardini, 2011: 19), dando origen a la "tesis Maisto", que enunció el embajador venezolano ante la Casa Blanca, según la cual Chávez debía ser juzgado por lo que hacía y no por lo que decía.

Sin embargo desde nuestra perspectiva, uno de los elementos más novedosos del ALBA es que al igual que su matriz chavista, hace una utilización sistemática del mito de Bolívar. Por cierto, esta lectura propuesta es subsidiaria de aquella que afirma que el mito político ocupa un lugar central en la orientación subjetiva hacia la política. Desde dicho punto de vista el mito político se estructura a partir de la gravitación que ejercen las narraciones e imágenes públicas acerca de acontecimientos y personas (los héroes) los que a su vez inciden sobre la conducta política por medio de la fascinación emocional. A partir de ello, procesos como la legitimidad política de un gobierno, la consolidación de una comunidad nacional y, por cierto, la integración que es el tema que nos ocupa, son dotados de sentido útil a las opciones de acción. Según Vuving (2009) el mito político es una herramienta que produce el poder blando en forma directa, junto a otros recursos como la promoción de la multilateralidad, la asistencia económica y el respaldo diplomático, además de otros instrumentos de producción indirecta de poder tales como los eventos culturales, la política comunicacional o la asistencia humanitaria, elementos también presentes en el ALBA. Como sabemos el concepto de poder blando fue acuñado como "la capacidad de afectar a los demás para obtener los resultados que se quieren a través de la atracción en lugar de la coerción o el pago" (Nye, 2004: 5). Es decir, frente a la amenaza militar o la recompensa económica, Nye coloca el poder blando con sus recursos culturales, los valores políticos y la propia política exterior de un Estado como mecanismos de poder (Nye, 2013: 566). Posteriormente, se ha puesto el acento en la persuasión ejercida hacia la política, la cultura o los ideales de un país (Montiel, 2010). Es decir, el poder blando es un factor altamente relevante en Estados pequeños y medianos con escasa significación militar y magro desempeńo económico (Landelius, 2012: 154). Respecto al tipo de atracción ejercido para Vuving (2010) puede ser comprendido como las monedas de la benignidad, la brillantez y la belleza. La benignidad es generada por medio de la gratitud y la empatía de compartir temas comunes, la brillantez apela al rendimiento en términos de admiración provocado por determinadas 
acciones y finalmente la belleza se refiere a la inspiración que despierta evocar la representación de ideales, valores o visiones. El bolivarianismo chavista con sus contenidos de reformas y de cambio, hace uso de este poder blando directo en su política exterior (de continuidad) hacia El Caribe o en sus relaciones preferenciales Bolivia y Ecuador en el marco de ALBA. Pero también la originalidad de los nuevos proyectos como TELESUR o las Operaciones Milagro, manifestaciones indirectas de este poder blando como se recoge la Tabla $\mathrm{N}^{\circ} 1$.

Tabla No 1: Bolivarianismo y poder blando indirecto

\begin{tabular}{|c|c|c|c|}
\hline $\begin{array}{l}\text { DISCURSO O } \\
\text { POLÍTICA }\end{array}$ & $\begin{array}{l}\text { CONTINUIDAD O } \\
\text { PROFUNDIZACIÓN }\end{array}$ & $\begin{array}{l}\text { REFORMA } \\
\text { GRADUAL }\end{array}$ & CAMBIO RADICAL \\
\hline $\begin{array}{l}\text { Rechazo al modelo } \\
\text { Neo-liberal } \\
\text { y a esquemas } \\
\text { controlados por } \\
\text { Estados Unidos }\end{array}$ & & & Oposición al ALCA \\
\hline Integración & $\begin{array}{l}\text { Relaciones privilegiadas con } \\
\text { el CARICOM (creación de } \\
\text { PetroCaribe) } \\
\text { Relaciones privilegiadas con } \\
\text { Bolivia y Ecuador } \\
\text { PBD (Poder blando directo) }\end{array}$ & $\begin{array}{l}\text { Creación de } \\
\text { PetroCaribe } \\
\text { PBD (Poder } \\
\text { blando directo) }\end{array}$ & $\begin{array}{l}\text { Creación del ALBA } \\
\text { Mito PBD } \\
\text { (Poder blando directo) }\end{array}$ \\
\hline & & & $\begin{array}{l}\text { TELESUR - PBI } \\
\text { (Poder blando directo) }\end{array}$ \\
\hline & & & $\begin{array}{l}\text { Ampliación de ciertas } \\
\text { políticas sociales más allá } \\
\text { de las fronteras nacionales } \\
\text { (Operación Milagro) } \\
\text { PBI (Poder blando } \\
\text { indirecto) }\end{array}$ \\
\hline
\end{tabular}

Fuente: Elaboración propia.

En ese sentido, aunque Venezuela ha practicado un particular culto a Bolívar, como nos recuerda Carrera Damas (1973), el mito político de la figura del prócer ha permitido llevar lejos las posibilidades de imaginar otros espacios / esquemas cooperativos, regidos por una lógica social solidaria (ideales), ensayando discursos alternativos al capitalismo, promoviendo forjar "el equilibrio del mundo" (valores empáticos), tarea que exige, primero, subvertir y transformar el orden económico y social (visiones) existente (Mora, 2010). Por cierto que es posible advertir diferencias entre el discurso bolivariano original, menos ocupado de temas sociales y sin visos de anticapitalismo, y el que esgrime el internacionalismo 
chavista. Sin embargo, lo relevante aquí es que este nuevo proyecto se inspira en el prócer (Romero y Quiñónez, 2010: 162) para cerrar un ciclo que se estima abierto e inconcluso desde las guerras de independencia: la emancipación social de los más pobres (Narvaja de Arnoux; 2008: 74).

Bajo esta impronta es que en 2004 se firmaron los acuerdos de La Habana. El ALBA se convirtió en una alternativa al ALCA. Al año siguiente el ALCA colapsa en Mar del Plata. Y recién en el año 2009, en la VI Cumbre Extraordinaria del ALBA, el presidente Hugo Chávez propone que se le denomine Alianza.

Los principios declarados fueron:

a) Comercio e inversión como medios para el desarrollo justo y sustentable, para lo cual se confió al Estado el rol regulador,

b) Trato especial y diferenciado a las economías más pequeñas mediante la cesión de ventajas,

c) Complementariedad económica y cooperación entre los suscriptores sin competencia,

d) Cooperación y solidaridad para países menos desarrollados,

e) Creación de un Fondo de Emergencia Social,

f) Desarrollo integrador de comunicaciones y transportes,

g) Compromiso con el desarrollo sustentable con apego a normas medioambientales, y uso racional de recursos,

h) Integración energética,

i) Fomento a la inversión al interior de la región,

j) Defensa cultural y de identidad de la región,

k) Normas equilibradas de propiedad intelectual que resguarden el patrimonio sin amenazar la cooperación,

l) Actuación multilateral concertada.

El gobierno boliviano de Evo Morales había adherido a dichos principios en abril de 2006. Complementariamente, y enmarcado en la lógica no libremercadista del intercambio compensado, fue firmado un Tratado de Comercio de los Pueblos (TCP), contrapunto conceptual hacia las negociaciones emprendidas por tres Estados miembros de la CAN (Colombia, Ecuador y Perú) para adoptar tratados bilaterales de libre comercio con Estados Unidos. En su lugar se propusieron un conjunto de acuerdo de comercio compensado, atendiendo a la idea de ventajas cooperativas antes que a lógica tradicional de ventajas competitivas (Briceño, 2011: 37), cuyo objetivo fue proveer de una mayor sustentabilidad económica al proyecto ALBA.

Pero más relevante, a los citados documentos fueron la traducción concreta del concepto "Diplomacia de los Pueblos", esbozada durante la contra-cumbre de Mar del Plata (Argentina), aquel mismo noviembre de 2005 referido, que aludía al rol específico 
de los movimientos sociales en los procesos descritos como una modalidad participativa (Sanahuja, 2009: 224-227). Desde este punto y simultáneamente al giro que experimentó la política exterior de Venezuela (Aranda, 2013:200-202) es posible consignar que la original "alternativa" al ALCA y la oposición a la prioridad de las premisas de liberalismo comercial impuestas a la región (Oyarzún, 2008:10) pasaron a constituirse en franco antagonismo a los valores políticos, económicos y sociales que Washington promueve en la región (Gardini, 2010: 14). Con ello ALBA reforzó su interés en desplegar mayores márgenes de autonomía geopolítica respecto de la potencia dominante.

Así entre 2004 y 2006 el ALBA se constituyó en un regionalismo multidimensional, aunque primordialmente político, en el cual la lucha contra la pobreza y el desarrollo social tienen especial atención (Quintanar, 2012:301). Entre sus rasgos constitutivos destaca la participación de un número limitado de países sin contigüidad geográfica, complementariedad económica sobre la competencia; la cooperación y solidaridad, la disminución de las asimetrías entre los Estados (Quintanar, 2012: 302). De esta manera, las economías menos desarrolladas de la región fueron tentadas por la posibilidad ofrecida por el ALBA y el socialismo del siglo XXI de una trasformación estructural que privilegiaría producción y valor agregado en sectores estratégicos sobre el comercio de materias primas (Muhr, 2011: 111). Lo anterior confirma que ALBA no es una propuesta basada en el comercio ni mucho reduce al intercambio comercial su proceso de integración (Briceño, 2011: 36), colocando en el centro del mismo la cooperación. Sobre dicho mensaje a los originales Cuba, Venezuela y Bolivia, se fueron agregando nuevos miembros: Nicaragua, Dominica, Honduras (que posteriormente se retiraría), Ecuador, San Vicente y las Granadinas y Antigua y Barbuda. Mientras tanto Haití, Paraguay, Grenada, Uruguay, El Salvador y República Dominicana se han mantenido, entre otros países, como observadores.

En consecuencia, se constituyó una agenda política de integración, de cooperación energética e intercambio económico dirigida por actores estatales (Sanahuja, 2009: 224). Este rol renovado fue también apuntalado por medio de la "Diplomacia de Cumbres", particularmente dinámica en sus primeros cinco ańos de vida (2004-2009), lapso de tiempo en que se verificaron quince encuentros en los que se definieron los objetivos aludidos y se perfiló su estructura, además de recibir a los nuevos miembros 5 . Constitucionalmente la democracia participativa y protagónica instaurada en la República Bolivariana de Venezuela no puede comprometer la soberanía nacional o transferir facultades a órganos supranacionales ni siquiera por la

\footnotetext{
${ }^{5}$ En 2009 se retiró Honduras y se ralentiza la velocidad de implementación de acuerdos. En 2010 se celebraron dos cumbres, el 2011 ninguna y en febrero de 2012 se retomó el impulso original en la IX Cumbre de Caracas, que sin embargo no continuó con el agravamiento de la condición de salud del presidente Chávez.
} 
vía de los referendos aprobatorios de los tratados (Viciano, 2010: 55). Este argumento es referido por algunos investigadores que aseguran que el ALBA es básicamente un proyecto político y una estrategia de cooperación sur-sur que no pretende integrar en un área económica a sus Estados miembros (Sanahuja, 2009:228). Otros investigadores como Gardini, en cambio, matizan dicha afirmación destacando el consenso de sus Estados parte en términos de la dirección política sobre la dimensión económica: "Es una reafirmación de lo político sobre lo económico y, a la vez, en un intento por recuperar un papel central para el Estado" (Gardini, 2010: 20).

En nuestra opinión ello significa que las metas sociales y económicas del ALBA están subordinados a la intergubernamentalidad del proyecto -noción que respondería al concepto teórico de soberanías compartidas previsto por Mitrany $(1970)^{6}$ - reflejada en la práctica concreta de una institucionalidad cuya cúspide es el Consejo Presidencial que reúne a los Jefes de Estados, quienes toman decisiones por unanimidad o consenso y bajo los cuales hay cuatro Consejos temáticos: a) Consejo Social destinado a los programas y proyectos sociales, b) Consejo Económico, que se ocupa de consolidar una zona de desarrollo compartido, con un Banco, integración energética y mecanismos de pago SUCRE (Sistema Único de Compensación Regional de Pagos), lo que apunta a un incipiente espacio económico (ECOALBA) c) Consejo Político, con la membresía de los ministros de relaciones exteriores que coordinan al bloque y finalmente d) El Consejo de Movimientos Sociales, dirigido a articular el respaldo de organizaciones sociales con la toma de decisiones y gestión del bloque, incluso más allá del ALBA a través de Escuela de Formación Política en países como Colombia, Perú y Chile.

Frente a este organigrama, Muhr sugiere cinco mecanismos funcionales para comprender el alcance de ALBA: a) acuerdos regionalistas entre Estados, b) instrumentos binacionales entre Venezuela y la mayoría de los países latinoamericanos, c) acuerdos multinacionales, d) acuerdos subregionales y e) instrumentos transnacionales no gubernamentales (Muhr, 2010: 5). Estos cinco mecanismos son cruciales para entender la naturaleza del ALBA-TCP ya que esta ética contra-hegemónica de solidaridad y cooperación se fusiona con lo históricoideológico en la construcción de un complejo "regional" y una "comunidad regional" "...y aportando las bases de un proyecto de gobernanza regional" (Absell, 2012: 80). En definitiva ALBA es parte de una estrategia de cooperación destinada a empoderar Latinoamérica y El Caribe y a des-hegemonizar la globalización capitalista, bajo un esquema poli-céntrico o multipolar, en nombre de naciones y pueblos, aunque dirigido principalmente por Estados, refrendando las presunciones que fundamentan el poder de los proyectos comunitarios básicamente en los

\footnotetext{
${ }^{6}$ Desde la perspectiva de Mitrany la soberanía compartida en vez de disolver a los Estados nacionales en unidades supranacionales, fortalecían ciertas condiciones estatales.
} 
Estados que los conforman y de los vínculos de estos con los grupos de interés de cada Estado (Taylor, 1996). El papel de Venezuela apunta al fortalecimiento de este tipo de cooperación en tanto condición imprescindible para establecer "polos de poder alternativos" a la hegemonía norteamericana (González, 2006) que posibilitarán una política exterior más independiente y capaz de viabilizar un proyecto de desarrollo ajeno a las premisas neoliberales. Lo anterior significa potenciar las alianzas con gobiernos y movimientos políticos afines o con grados de autonomía respecto de Washington ${ }^{7}$. Surge entonces un nuevo momento regional que se distingue del anterior por su evaluación positiva en retrospectiva del carácter estratégico del regionalismo de mediados de siglo XX (particularmente en empresas de áreas estratégicas que son sustraídas de la pura lógica de mercado), con un sello multidimensional, aunque sin prioridad en lo económico, y con políticas aglutinadas en torno del concepto de soberanía compartida y a la actuación geopolítica colectiva regional (Aponte-García, 2013: 16).

En esta práctica, los Estados aparecen nuevamente como portadores de un proyecto de integración que no los tiene como fin en sí mismos -al declararse la relevancia de la integración de pueblos y naciones-, aunque lo protagonizan mediante un activo intergubernamentalismo. Este se expresa en la rúbrica de acuerdos presidenciales y la confección de programas estratégicos que detallan las acciones concertadas y específicas a que cada Estado se compromete (Espinosa, 2011: 180). Dicho proceso pretende incidir en la distribución de la riqueza mediante un programa de cooperación en áreas sociales sensibles como es la construcción de viviendas, combate al analfabetismo y provisión de salud, jugando Cuba un papel primordial respecto de dichos tópicos ${ }^{8}$. Así la redistribución de la riqueza es parte consustancial a la inclusión. A lo anterior se agregan otras iniciativas como Petro Caribe, el Banco del Sur y TELESUR.

Finalmente, ALBA reconoce el derecho social de los pueblos para definir sus prioridades agrícolas y alimentarias, lo que implica defender dicha área de las fluctuaciones de precios provocadas por el libre comercio. Precisamente, respecto a este punto ALBA pretende reducir considerablemente las asimetrías entre países por medio de la creación de sistemas alternativos al comercio internacional. Su lema es que el libre comercio entre desiguales termina por favorecer a los más poderosos, por lo que se propone un comercio justo y tratamiento especial y diferenciado, a través del principio de no reciprocidad y comercio compensado, sobre el cual

\footnotetext{
${ }^{7}$ Lo anterior se vierte en decisiones de Caracas tales como relegar a un segundo plano los ámbitos tradicionales de integración del país (la Comunidad Andina o el Grupo de los Tres) y dar un "giro hacia al sur" mediante el ingreso como miembro pleno del Mercado Común del Sur en 2012.

${ }^{8}$ El presidente Chávez aseguró en Mar del Plata que "El ALBA gracias al Acuerdo Estratégico CubaVenezuela ha podido derrotar un mal de siglos: el analfabetismo”. Véase Chávez (2006: 268).
} 
podemos ejemplificar con el intercambio de petróleo por prestaciones médicas. Las nociones de soberanía compartida, propia del intergubernamentalismo, y la cooperación internacional (Espinosa, 2011: 163) ocupan el lugar del libre comercio en la constitución de una entidad subregional dotada de mayores márgenes de autonomía en la voluntad de proyectar su propia voz sobre el nivel global.

\section{LA ALIANZA DEL PACÍFICO}

La emergencia del ALBA expuso una alternativa al discurso hegemónico de integración regional característico de los noventa, aunque también supuso el fin de la convergencia que la región exhibió respecto del derrotero de desarrollo. En los extremos de los modelos de inserción latinoamericana al empezar el siglo XXI habíamos tenido, por un lado, "la inserción plena a la economía mundial y por otra, la especificidad política nacional que influyen de forma decidida en los distintos objetivos nacionales y establece las condiciones de desarrollo dentro de los procesos de integración" (Palomares, 2004: 162). Sin embargo, aún podemos reconocer la tendencia a que persistieran opciones de inserción internacional liberal, como en el caso de Chile, Colombia y Perú, mientras aquella sustentada por Venezuela, Bolivia y Ecuador ensayó el citado regionalismo multidimensional de fuerte sello político emancipador (Alzugaray, 2009) con carácter social y geopolítico (Giacalone, 2008: 26).

El regionalismo de fines de los noventa había sido animado por el concepto de regionalismo abierto. Este alude al contexto del fenómeno de la interdependencia y de la cooperación económica de los países de la región de Asia Pacífico en la década del setenta del siglo XX. Dicho término, se refiere hoy a las acciones que están orientadas a incrementar la interdependencia entre los países de una región ${ }^{9}$, en el marco de una tendencia sostenida hacia el libre flujo de los factores productivos. Se distinguió del regionalismo cerrado, por una vocación manifiesta a la negociación comercial con otros mercados extra-regionales en una malla de acuerdos que se superponen, siempre abiertos a la potencial adhesión de nuevos participantes. Por lo tanto, evita la práctica de estructuración de mercados regionales protegidos, que adopta mecanismos de autodefensa frente a un Orden Mundial percibido como cambiante y hostil a los intereses de sus unidades componentes.

\footnotetext{
${ }^{9}$ Si bien el regionalismo abierto busca la integración regional sin reducir la interdependencia con el resto del mundo, también se refiere también a la noción de región basada en el reconocimiento de una experiencia histórica común y un sentimiento compartido de problemas en sociedades geográficamente identificadas. Lo anterior posibilitaría la creación de vínculos con cierto grado de intensidad mayor de contactos con respecto a otras áreas del resto del mundo.
} 
Pero aún más si seguimos a Sunkel el que llamamos "neo regionalismo" trataba de vincular el desarrollo nacional al incremento del intercambio comercial, así como el flujo económico y financiero latinoamericano en el marco del regionalismo abierto (Sunkel, 1998). De esta manera, la convergencia económica intrarregional no fue vista como óbice a la convergencia económica interregional. La categoría más sobresaliente a este respecto es el proceso de integración económica regional, que se expresa en la profundización y organización de la interdependencia económica en una región, sin descuidar las relaciones económicas con otros países $\mathrm{y}$ regiones del mundo.

Según sostuvo Van Klaveren (1998: 126) el regionalismo abierto expresó tres alcances. Primero, las distintas opciones de inserción regional no son apreciadas como mutuamente excluyentes, sino que tienden a complementarse. Así, la inserción internacional múltiple se traduce en plantear, por ejemplo, una no incompatibilidad entre las vinculaciones con América Latina, la Unión Europea y Asia. Segundo, los acuerdos están abiertos a la incorporación de nuevos miembros. Y tercero, la profundización de los esquemas regionales pretende hacerse compatible con la liberalización del comercio global, evitando, por lo tanto, el surgimiento de nuevas barreras en torno a los bienes y servicios que provienen desde fuera de la región.

La dinámica del regionalismo abierto fue asumida por CEPAL (1994), recomendando preferencias intrarregionales que simultáneamente no descuidarán el grado de apertura necesaria al intercambio global. Esta mirada proponía que la vinculación con la región de América Latina correspondía a estrategias nacionales de inserción política y económica de carácter global, explicadas en el interés de promover un entorno regional estable para el comercio. Estados como Chile e incluso Brasil implementaron con diverso resultado dicha estrategia, que solo en el siglo XXI pareció insuficiente, por cuanto no daba respuestas a otras tendencias y desafíos, como la de una acción afirmativa de los Estados y sociedades para superar la dependencia de exportación de productos primarios, creación de capacidades de innovación, mayor vínculo de lo político, económico y científico-tecnológico, incorporación de la variable cultural y las demandas sociales de equidad en los niveles de desarrollo.

El problema que una vez suscritos gran cantidad de TLC y tras sucesivas crisis del modelo de apertura comercial consensuada, este regionalismo abierto comenzó a exponer claros síntomas de agotamiento. En dicha coyuntura apareció primero el Arco del Pacífico en 2007, congregando a once países latinoamericanos ribereños al Pacífico, interesados en promover el comercio conjunto con Asia. Sin embargo, ciertos participantes estuvieron más comprometidos que otros en la apertura comercial extra-regional, proyectándose otro acuerdo más acotado solo con aquellos miembros que coincidieran en la visión económica aperturista 
y la plena liberalización de los factores comerciales. El resultado fue la Alianza del Pacífico, en abril de 2011, en que los Estados de la región con economías más liberales -y adicionalmente con similares niveles de desarrollo (Ravenhill, 2011)_ se comprometieron a alcanzar mayores rangos de coordinación cooperativa para acceder a la macro-región del Asia-Pacífico.

La Alianza del Pacífico originalmente reunió a Colombia, Chile, México y Perú, quedando como candidatos observadores Panamá y Costa Rica, en un serio intento de preservar el modelo aperturista, que les había reportado claros beneficios mediante la asociación en una zona de libre comercio amplia y coordinada. Con apenas tres años de vida ha organizado IX cumbres presidenciales y suma nuevos observadores oficiales con cerca de treintados Estados en dicha calidad. Aunque claramente en las antípodas ideológicas del ALBA, heredó del anterior la escasa atención a ciertas premisas de la teoría clásica de integración, por ejemplo, respecto de la contigüidad geográfica, aunque en este caso la voluntad de "cooperar para salir juntos al mercado" se explicaba en el interés de no renunciar a los réditos económicos producido por el crecimiento experimentado en la última década y media. Si el ALBA mostró la prioridad de lo político sobre lo económico la Alianza del Pacífico mantuvo intacta la primacía económica incluso entre Estados con contenciosos fronterizos (Chile y Perú), mediante la libre circulación de los factores de producción (bienes, servicios, capitales y personas) aunque con una importante novedad: La cooperación se irguió junto a la característica competencia del mercado como un rasgo constitutivo del acuerdo signado durante la presidencia pro témpore de Chile, en junio de 2012.

La apuesta pareció reforzarse ante la eventual futura incorporación de países como Costa Rica (iniciando el proceso de adhesión en febrero de 2014), probablemente en el futuro Panamá, motivados por el dinamismo de las economías miembros cuya suma representa el 35\% del PIB regional latinoamericano, y un comercio que en su conjunto equivale al 55\% del total regional. Adicionalmente se promovió la implementación de un paquete de medidas en el "Memorando de Entendimiento para la Plataforma de Cooperación de la Alianza del Pacífico", que prevé la colaboración en áreas medioambientales, innovación, ciencia y tecnología, empresas y desarrollo social. Adicionalmente se ha promovido el establecimiento de misiones conjuntas o coordinadas -incluso oficinas de representación- con el Asia Pacífico y la armonización de las reglas de origen en aspectos aduaneros específicos, lo que ha ido generando convergencias más amplias, facilitadoras, por ejemplo, de la integración de los mercados financieros de Bogotá, Lima y Santiago (Silva, 2012b: 6). Durante la última cumbre de febrero de 2014, los Estados parte se comprometieron a colaborar en asistencia consular conjunta en situaciones de emergencia. Todo lo anterior apunta a la revitalización en la Alianza del Pacífico del espíritu de regionalismo abierto típico a fines del siglo anterior. A lo 
mencionado se agrega la facilitación del movimiento general de personas entre los Estados fronterizos y en menor medida para México (López y Muñoz, 2012: 21). En esta área se pretende establecer una plataforma de movilidad académica que contribuya al fortalecimiento de capital humano avanzado de los países signatarios.

Flexibilidad y capacidad de ejecución reposa en su estructura intergubernamental -como el ALBA- fundamentalmente técnica y a lo sumo ministerial (Grupo de Alto Nivel encargado de evaluar los avances en las diversas materias, el Consejo de Ministros encargados de adoptar decisiones específicas, los Grupos Técnicos abocados a las negociaciones en temas específicos y, por el último, el Consejo Empresarial). Las decisiones, al igual que el ALBA, provienen de autoridades centrales del Estado que retienen todo el control de las políticas, decisiones y actividades de la organización, con la diferencia que el actor invitado en el caso anterior son los movimientos sociales y el empresariado en la Alianza del Pacífico.

\section{CONCLUSIONES}

ALBA y Alianza del Pacífico son parte de dos esquemas de cooperación con diferencias ideológicas, pero también con elementos comunes. Coincidentemente ambas propuestas no se ciñen al credo funcionalista de integración y al modelo arquetípico de la experiencia de la Unión Europea. Criterios como la proximidad geográfica o el "desborde" por áreas no son requisitos para ninguno de los dos casos. Lo anterior explica que ambas alianzas sean heterogéneas respecto a su composición, superando el horizonte sudamericano para afincarse en Latinoamérica. EL ALBA tiene adherentes que participan en diversos acuerdos subregionales: MERCOSUR, CAN, SICA y CARICOM, mientras que la Alianza de Pacífico tiene miembros que son parte de la CAN y el NAFTA, y con postulantes del SICA.

El ALBA sitúa en el núcleo de su propuesta de integración la deuda social, lo que le permite desplegar recursos de poder blando directo (asistencia económica en programas energéticos) e indirecto (estación de televisión y asistencia humanitaria). En definitiva, se trata de un proyecto político y social y solo en segundo lugar de complementariedad económica. En este ámbito, el ALBA desea promover el comercio justo, eliminando asimetrías para alcanzar un equilibrio entre las partes y desde allí construir un camino cooperativo de una unidad que no se encuadra con el sistema dominante (Briceño, 2013: 16), aunque en nuestra opinión el discurso antagónico respecto del capitalismo no alcanza para la secesión sistémica como expone la preservación de la relación comercial con Estados Unidos por parte de sus miembros. En este sentido, uno de los principales mecanismos para la cooperación no liberal, son los Tratados de Comercio de los Pueblos que pretenden alcanzar el desarrollo 
conjunto mediante la diversificación del aparato productivo (Briceño, 2013: 34), blindando a los Estados del ALBA con sustentabilidad económica. Adicionalmente, la Alianza Bolivariana ha potenciado la diplomacia social por medio de la cooperación en temas de salud, educación y vivienda. La construcción de un espacio de protección respecto del mercado ha incidido en cierto margen de autonomía relativa de bloque y el cultivo de un alto perfil contestatario al sistema internacional.

En tanto que la Alianza del Pacífico responde al modelo de regionalismo abierto, aunque renovándolo mediante el reemplazo de la competencia por la cooperación en el acceso a los mercados de Asia-Pacífico. Dicha cooperación tiene su origen en el interés común por corregir conjuntamente fallas de mercado, explicando la interacción incluso en áreas que los TLC bilaterales no exigían, por ejemplo la colaboración en materia consular. La originalidad está sobre todo en la voluntad de coordinar posiciones comunes para acceder a los mercados de AsiaPacífico, aunque sin abandonar su compromiso con la región como testimonian becas y pasantías dirigidas a los ciudadanos de sus países, en una clara política de creación de confianzas mutuas por medio de la educación y la cultura. Es decir, aunque desde sus inicios ha tenido una agenda claramente comercial en torno al libre movimiento de bienes, servicios, capitales y personas, gradualmente ha diversificado su agenda, legitimando socialmente su viabilidad más allá del mercado.

Ambos, ALBA y Alianza del Pacífico, parecen aún insuficientes si se les mide desde la teoría clásica o se les confronta con resultados más ambiciosos, sin embargo, su propia experiencia los está conduciendo por derroteros que desbordan

el diseño original acercándose a una forma de integración no convencional, sin olvidar que esta integración más que declararse se recorre.

\section{REFERENCIAS}

Absell, Christopher. "El ALBA-TCP mirado con buenos ojos", Revista Problemas del Desarrollo, 169/43, abril-junio, (2012): 73-95.

Actis, Esteban. "Brasil y sus pares sudamericanos de la "Alianza del Pacífico". Divergencias regionales en las opciones nacionales e internacionales", Revista Relaciones Internacionales, 23/46, enero-junio, (2014): 71-87.

Alzugaray, Carlos. Los cambios en la agenda regional hemisférica ante los nuevos escenarios en América Latina y el Caribe y Estados Unidos. Ponencia presentada en Conferencia Internacional celebrada en Sala del Consejo Universitario de la Rectoría Universidad Estatal de Sao Paulo, 2009. 
Aponte-García, Maribel. The New Strategic Regionalism in ALBA-TCP: Alternatives to the food and energy crisis. (2012) Disponible en: http:// cicia.uprrp.edu/PII/aponte-pii2012.pdf.

Aranda, Gilberto. El Proyecto Chávez (1999-2007). Participación. Isocracia e integración regional. Santiago de Chile: Editorial Universitaria, 2013.

Balassa, Bela. Teoría de la Integración Económica. México: Biblioteca Uteha de Economía, 1964.

Bernal-Meza, Raúl. "El regionalismo: Conceptos, paradigmas y procesos en el sistema internacional contemporáneo". En Lincoln Bizzozero y Isabel Clemente (coords.), La Política Internacional en un mundo en mutación. Montevideo: Ediciones de la Banda Oriental, (2001): 11-30.

Bernal-Meza, Raúl. "Heterodox Autonomy Doctrine: Realism and purpose, and its relevance", Revista Brasileira de Política Internacional 56/2 (2013): 45-62.

Briceño, José. "El ALBA como propuesta de integración regional". En Josette Altmann Borbón (ed.), ALBA ¿Una nueva forma de Integración Regional? Buenos Aires: Editorial Teseo-FLACSO-Argentina, 2011.

Briceño, José. "Ejes y modelos en la etapa actual de la integración económica regional de América Latina”, Estudios Internacionales 175, Santiago, mayoagosto, (2013): 9-39.

Busso, Anabella. "Los vaivenes de la política exterior argentina re-democratizada (1983- 2013). Reflexiones sobre el impacto de los condicionantes internos", Estudios Internacionales 177, Santiago, enero-abril, (2014): 9-34.

Carrera Damas, Germán. El Culto a Bolivar. Esbozo para un Estudio de las Ideas en Venezuela. Caracas: Universidad Central de Venezuela, 1973.

Casilda, Ramón. "América Latina y el Consenso de Washington", Boletín Económico de ICE $\mathrm{N}^{\circ} 2803$ (2004): 19-38.

Espinosa, Eugenio. "El ALBA: Teoría y práctica de la integración regional”. En Josette Altmann Borbón (ed.), ALBA ¿Una nueva forma de Integración Regional? Buenos Aires: Editorial Teseo-FLACSO-Argentina, 2011. 
Gardini, Gian Luca. "Proyectos de Integración Regional Sudamericana: Hacia una teoría de convergencia regional”, Revista Relaciones Internacionales 15 (2010): 11-31.

Gardini, Gian Luca. "Latin America Foreing Policy between Pragmatism and Idelogy: A framework for Analysis". In Gian Luca Gardini y Peter Lambert. Latin America Foreing Policy between Pragmatism and Idelogy. New York: Palgrave MacMillan, 2011.

Giacalone, Rita. La integración sudamericana: Un complejo proceso inconcluso. Caracas: Universidad de Los Andes, 2008.

González, Edmundo. "Las dos etapas de la política exterior de Chávez", Nueva Sociedad 205/165 (2006): 159-171.

Hernández, Beatriz. A diez años del Acuerdo de Asociación UE-Chile: ¿El vaso medio lleno o medio vacío? En Después de Santiago: Integración regional y relaciones Unión Europea - América Latina. Coral Gables, Universidad de Miami, (2013): 117-131.

Hettne, Bjorn and Söderbaum, Fredick. "Theorizing the rise of regionness", New Political Economy 5/3 (2000): 457-473. Department of Peace and Development Research; Göteborg University, Sweden.

Hurrell, Andrew. On Global Order: Power, Values, and the Constitutions on International Society. New York: Oxford University Press, 2007.

Index of Economic Freedom, Heritage Foundation, 2006-2014, Washington, 2014.

Landelius, Peter. "Poder Suave y Diplomacia Pública en el contexto multilateral", Revista Mexicana de Politica Exterior 96 (2012): 151-168.

López, Dorotea y Muñoz, Felipe. "El inicio formal de la Alianza del Pacífico", Revista Puentes 13/4. Santiago de Chile: Universidad de Chile (2012): 18-22.

Montiel, Edgar. "Diplomacia Cultural. Un Enfoque Estratégico de Política Exterior para la Era Intercultural". Cuadernos UNESCO Guatemala 2 (2010):1-26. Disponible en: http://unesdoc.unesco.org/images/0019/001906/190623s.pdf. 
Malamud, Andrés; "Divergencias en ascenso: Viejas y nuevas fracturas en América Latina" en Araucaria, Revista Iberoamericana de Filosofia, Política y Humanidades, 21 (2009): 125-139. Disponible en: http://alojoptico.us.es/ Araucaria/nro21/monogr21_2.pdf.

Mitrany, David. "The functional approach to World Organisations". In Carol Anne Cosgrove y Kenneth J. Twitchetm (eds.), The new international actors: The UN and the CEE. London: Macmillan, 1970.

Mora, Andrés. "El equilibrio del mundo y la nueva integración latinoamericana". América Latina en Movimiento. Agencia Latinoamericana de Información (2010). Disponible en: http://connuestraamerica.blogspot.com/2010/04/ el-equilibrio-d el-mundo-y-la-nueva.html.

Morandé, José. "Globalización y regionalismo en el nuevo escenario internacional"; mimeo, Fundación Felipe Herrera Lane, 1999.

Muhr, Thomas. "TINA go home: Alba and the re-theorizing resistance to global capitalism". In Cosmo and History: The Journal and Natural and Social Philosophy, 5/2 (2010): 27-54. Disponible en: http://cosmoandhistory.org/ index.php/jounal/article/view/210.

Muhr, Thomas. "The ALBA-TCP. En Latin American Trading Blocs: Between reality and utopia", Institute of Advance Legal Studies (IALS) 15/33 (2011): 116-117.

Narvaja de Arnoux, Elvira. El discurso latinoamericanista de Hugo Chávez. Buenos Aires: Editorial Biblos, 2008.

Nolte, Detlef. "Potencias regionales en la política internacional: Conceptos y enfoques de análisis". Programa de Investigación. Dinámicas de violencia y cooperación en seguridad, $\mathrm{N}^{\circ} 30$. Instituto Alemán de Estudios Globales y Regionales. Disponible en: http://www.giga-hamburg.de//de/system/files/ publications/w30_nolte.pdf.

Nye, Joseph Jr. Soft Power: The Means to Success in World Politics. USA: Cambridge Center. 2004.

Nye, Joseph Jr. "Hard, Soft and Smart Power". In Andrew Cooper, Jorge Heine y Ramesh Thakuer (eds.), Oxford Handbook of Modern Diplomacy. Oxford: Oxford Press, 2013. 
Palomares Lerma, Gustavo. Relaciones Internacionales en El Siglo XXI. Madrid: Tecnos, 2004.

Payton, Anthony. "Repositioning the Commonwealth Caribbean". In Andrew Cooper y Jorge Heine (ed.), ¿Which way Latin America? Tokyo, New York, Paris: United Nations University Press, 2009.

Pope, Atkins. América Latina en el Sistema Político Internacional. Buenos Aires: Grupo Editor Latinoamericano, 1991.

Puig, Juan Carlos, “Introducción”. En Juan Carlos Puig. América Latina: Políticas exteriores comparadas. Buenos Aires: Grupo Editor Latinoamericano, 1984.

Oyarzún, Lorena. "Sobre la naturaleza de la integración regional. Teorías y debates", Revista de Ciencia Politica 28/3 (2008): 95-113.

Quintanar, Silvia. "Convergencias y divergencias en las estrategias de integración y cooperación energética regionales de Venezuela y Brasil”. En Raúl Bernal-Meza y Silvia Quintanar (eds.), Regionalismo y Orden Mundial: Suramérica, Europa, China. Buenos Aires: Grupo Editor Latinoamericano, (2012a): 241-281.

Quintanar, Silvia. "La Alianza Bolivariana para los Pueblos de Nuestra América (ALBA). Un nuevo proceso de regionalismo latinoamericano". En Raúl Bernal-Meza y Silvia Quintanar (eds.), Regionalismo y Orden Mundial: Suramérica, Europa, China. Buenos Aires: Grupo Editor Latinoamericano, (2012b): 301-321.

Ravenhill, John. "Regional Trade Agreements". In John Ravenhill (ed.), Global Political Economy. Oxford: Oxford University Press, 2012.

Romero, Juan Eduardo y Quińónez, Yessica. "El pensamiento socio-político de Chávez: Discurso, poder e historia”, Cuadernos Latinoamericanos 38 (2010): 19-38. Maracaibo. Centro Experimental de Estudios Latinoamericanos Dr. Gastón Parra Luzardo, Universidad de Zulia.

Sanahuja, José Antonio. "La integración regional, los proyectos bolivarianos, y la Unión de Naciones Sudamericanas". En Vicente Garrido (ed.), Venezuela y la Revolución Bolivariana. Madrid: Instituto Universitario General Gutiérrez Mellado de Investigación la Paz, la Seguridad y la Defensa, (2009): 201-250. 
Silva, María Cristina. "Implicancias de la Integración regional latinoamericana de inicios del siglo XXI". Trabajo preparado para su presentación en el X Congreso de Ciencia Política. Santiago de Chile, octubre, 2012.

Sunkel, Osvaldo. "Desarrollo e Integración. ¿Otra oportunidad para una promesa incumplida? Revista CEPAL, edición especial, octubre. Santiago de Chile: CEPAL, (1998): 229-241.

Taylor, Paul. The European Union in the 1990's. Oxford: Oxford University Press, 1996.

Van Klaveren, Alberto. "Inserción internacional de Chile". En Cristián Toloza y Eugenio Lahera (eds.), Chile en los noventa. Santiago de Chile: Dolmen Editores y Presidencia de la República, (1998): 117-160.

Viciano, Roberto. "La columna vertebral de la revolución: el fenómeno participativo en la Venezuela Bolivariana”. En Juan Torres López (ed.), Venezuela, a contracorriente. Los origenes y las claves de la revolución bolivariana. Barcelona: Icaria y Madera Editorial, 2010.

Vuving, Alexander. "How Soft Power Works", American Political Science Association Annual Meeting (2009): 1-20. 